\title{
Method of Diagnosis and Treatment of Impacted Teeth on the Upper Jaw
}

\author{
MA Postnikov*, DA Trunin, MM Kirilin and MV Chistyakova \\ Samara State Medical University, Russia
}

*Corresponding author: MA Postnikov, Samara State Medical University, Russia.

Received Date: January 22, 2020

Published Date: February 13, 2020

Abstract maxilla.

Objectives: To develop and propose a new technique for determining the topography of impacted teeth (incisors and canines) on the maxilla, using the highly informative data of cone-beam computed tomography; to apply a new method of diagnosis in the planning of comprehensive orthodontic treatment of patients with impacted teeth; to introduce this method into the clinical practice of orthodontist.

Materials and methods: To obtain a three-dimensional image, the X-ray device "Planmeca Pro Max 3D Mid" with «Planmeca Romexis»-the software for saving, viewing and changing images was used. To build and conduct measurements on the image the program "Dolphin Imaging" (3D Surgery and Ceph Tracing) (USA) was used. A computed tomogram of patient was used to determine the levels of location of impacted teeth and angles of their longitudinal axes inclination in three mutually perpendicular planes according to the proposed technique.

Results and summary: A technique of determining the spatial location of the frontal impacted teeth on the maxilla was developed and introduced into practice of the orthodontist, using data from cone-beam computed tomography. The proposed method allows to determine the level of location and degree of its inclination, that is very important for planning comprehensive orthodontic and surgical treatment of patients with impacted teeth.

Keywords: Impacted teeth; Diagnosis; Orthopantomography; Cone beam computed tomography; Comprehensive orthodontic treatment

\section{Relevance}

Diagnosis and treatment of patients with impaction of permanent teeth is one of the urgent problems of modern dentistry due to morphological, functional and aesthetic changes that occur in case of absence of a tooth in the dentition, which leads to violation of the social adaptation of the individual. Today, tooth impaction is a fairly common anomaly of the maxillofacial system: for 100 children seeking orthodontic care, 15-20 have malocclusion complicated by the impaction of one or more teeth (Budkova TS, Zhigurt Yu I, Khoroshilkina FI, 1997). The front teeth of the upper jaw are more often impacted: Central incisors and canines-61.6\% (Stepanov GV, 2008).

In classical cases of treatment of patients with impacted teeth, traditional orthodontic treatment should be chosen $[1,2,3]$.
However, in cases when the impacted tooth has an anomaly of location, improper inclination or unfavorable individual anatomical features, undoubtedly, combined surgical and orthodontic treatment is preferable $[2,4,5]$. It is here that the determination of the exact location of the retained tooth is especially important. When teething is delayed, X-ray examination methods are fundamental both in the diagnosis and in the treatment planning.

There are some methods of determining the location levels of impacted anterior teeth. The most common and generally accepted is the method proposed by Yu I Zhigurt [6]. The study of the impacted teeth location conducts using orthopantomogram of jaws, where 4 levels of location the impacted upper incisors and canines are determined. At the orthopantomogram we draw a median line, which coincides with the median plane. Then, 2 horizontal lines are 
drawn perpendicular to the median plane: one-through the point of the prostion, the second-through the point of the anterior nasal spine. The vertical distance between these lines is divided into 4 equal parts and horizontal lines are drawn through the obtained points. The first level of tooth location is between the two lower lines, the fourth is between the two upper lines, the second and third are in the middle [6].

Method of Yu I Zhigurt [6] can also be used to measure the angle of inclination of the impacted teeth: the mid-sagittal plane is drawn on the orthopantomogram through the middle of the vomer and the anterior nasal spine. Then we draw a horizontal plane perpendicularly to level of incisal surface of the permanent central incisors of the upper jaw. Angles of inclination of the longitudinal axes of impacted teeth located in the anterior part of the dentition (upper internal angles) are determined relative to this plane. Three degrees of inclination of the longitudinal axes of the impacted teeth were determined: 1 degree-up to $105^{\circ}, 2$ degree-105-120, 3 degree-more than $120^{\circ}$ [6].

Despite the popularity among orthodontists, this method has a number of significant drawbacks. It is impossible to reliably determine the position and placement of the elements of the upper and lower jaw at the orthopantomogram, including teeth, since the anatomical structures are deformed due to the layering them on each other. It was also established that the true vertical and horizontal linear dimensions of teeth and jaws are significantly distorted at the orthopantomogram. As a result, it becomes impossible to determine the overall magnification of the image. In addition, at the orthopantomogram we perform a slice at a certain depth, therefore, the image of impacted teeth located not in the center of the alveolar bone can be either enlarged or reduced (Khoroshilkina F Ya, 2006). All of the above allows us to say that this method is not sufficiently informative and does not allow us to accurately assess the location of impacted tooth in the jaw [7].

\section{Aim of study}

To increase the efficiency of diagnostics and comprehensive treatment of patients with impacted teeth by creating a new method of determining the location of impacted maxillary incisors and canines, using cone beam computed tomography.

\section{Materials and Methods of Study}

The development of method of diagnosis and comprehensive treatment of patients with impacted maxillary incisors and canines was carried out at the department of postgraduate dentistry of Samara state medical university. 12 computed tomograms of 6 patients with impacted maxillary teeth were obtained and studied. Each patient corresponded to 2 three-dimensional images, one of which was used to study the level of location, and the second oneto determine the angle of inclination of the tooth longitudinal axis.

We used x-ray unit «Planmeca ProMax 3DMid» for cone-beam computed tomography. We used computer graphics software «Planmeca Romexis» to work with the x-ray images. After performing a CBCT in the area of the impacted tooth, an X-ray image was obtained in three planes: coronary-left upper corner, axial-left lower corner, sagittal-right upper corner, and volumetric imageright lower corner, which allows you to determine the current orientation X, Y and Z planes and current image scale. There is a toolbar on the right. A rectangular coordinate system was displayed in the window of each slice, with the help of which the parameters of the impacted tooth and its location in the jaw were measured [8].

We used the 3D module of the Dolphin Imaging computer program (USA) to determine the level of location of the impacted teeth and to take measurements on the obtained image. The method we have proposed includes determining the location of levels of the impacted maxillary frontal teeth (incisors, canines) according to cone beam computed tomography. We draw two horizontal lines on the patient's CT scan: one through the points of Spina nasalis anterior (Sna) and Spina nasalis posterior (Snp), and the second through the apical basis of the upper jaw. We divide the vertical distance between these lines into three equal parts and draw horizontal lines through the resulting points. The first level of tooth location is between two lower lines, the third is between two upper lines, the second is in the middle (Figure 1).

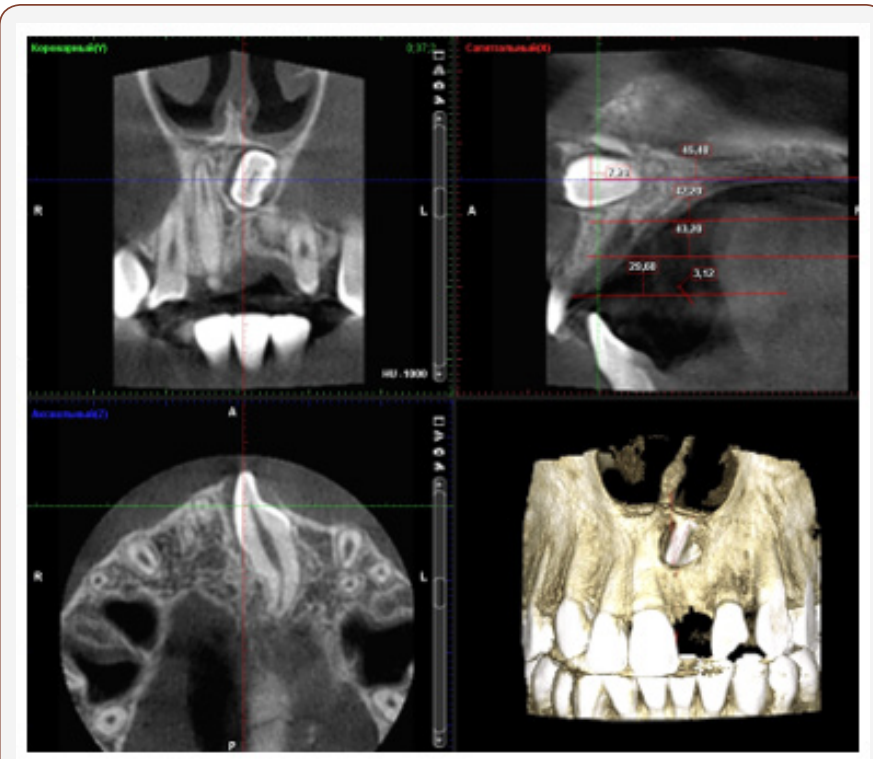

Figure 1: Determination of three levels of the impacted teeth location (incisors and canines of the upper jaw) on the CBCT of patient A, 14 years old (III level of tooth 2.1 location).

To determine the level of location of the impacted tooth on the CBCT, an image in the sagittal plane was used. It was found that it is advisable to distinguish precisely three levels of location of the impacted tooth, since the vertical distances between the horizontal lines correspond to the size of crown of the impacted tooth. We determine the level of location of the impacted tooth by the zone, where tooth's crown is. There is a rectangular coordinate system in the window of each plane on the CBCT. The axes of this system were used by us to measure the angles of inclination of the impacted teeth axes [9].

To measure the axis of inclination of the impacted tooth, we measured the angle, one side of which was the middle axis of the 
impacted tooth, and the other side was a line drawn parallel to the horizontal axis of the rectangular coordinate system in the sagittal plane and parallel to the vertical axis of the coordinate system in coronary and axial planes. The angle was determined with an accuracy of $0.0^{\circ}$. According to our method, 3 degrees of inclination of the impacted tooth axes were determined by computed tomogram in each of the planes (Table 1).

Table 1: Determining the degree of inclination of the impacted teeth axes (incisors, canines of the upper jaw) in three planes according to cone beam computed tomography.

\begin{tabular}{|c|c|c|}
\hline \multirow{2}{*}{ Plane } & Degree & Angle value \\
\hline \multirow{3}{*}{ Coronary } & 1 & up to $45^{\circ}$ \\
\cline { 2 - 3 } & 2 & $45^{\circ}-90^{\circ}$ \\
\cline { 2 - 3 } & 3 & more than $90^{\circ}$ \\
\cline { 2 - 3 } & 1 & up to $100^{\circ}$ \\
\cline { 2 - 3 } & 2 & $100^{\circ}-120^{\circ}$ \\
\hline \multirow{3}{*}{ Sagittal } & 3 & more than $120^{\circ}$ \\
\cline { 2 - 3 } & 1 & up to $45^{\circ}$ \\
\cline { 2 - 3 } & 2 & $45^{\circ}-90^{\circ}$ \\
\cline { 2 - 3 } & 3 & more than $90^{\circ}$ \\
\hline
\end{tabular}

\section{Research Results and Discussion}

Table 2: The degrees of inclination of the impacted teeth axes according to cone beam computed tomography.

\begin{tabular}{|c|c|c|c|c|c|c|}
\hline \multirow{2}{*}{ Patient } & \multicolumn{6}{|c|}{ Plane } \\
\cline { 2 - 7 } & Coronary & Degree & Sagittal & Degree & Axial & Degree \\
\hline 1 & $36,87^{\circ}$ & 1 & $118,72^{\circ}$ & 2 & $37,72^{\circ}$ & 1 \\
\hline 2 & $12,51^{\circ}$ & 1 & $105,39^{\circ}$ & 2 & $29,85^{\circ}$ & 1 \\
\hline 3 & $34,20^{\circ}$ & 1 & $136,45^{\circ}$ & 3 & $33,76^{\circ}$ & 1 \\
\hline 4 & $22,78^{\circ}$ & 1 & $175,25^{\circ}$ & 3 & $18,46^{\circ}$ & 1 \\
\hline 5 & $29,48^{\circ}$ & 1 & $141,09^{\circ}$ & 3 & $29,75^{\circ}$ & 1 \\
\hline 6 & $56,33^{\circ}$ & 2 & $129,88^{\circ}$ & 3 & $57,93^{\circ}$ & 2 \\
\hline
\end{tabular}

We conducted 12 computed tomograms of 6 patients with impacted maxillary teeth in the frontal part (incisors, canines) before treatment and found 6 impacted teeth (2 central incisors, 1 lateral incisor, 3 canines) located at different levels. First level of location was detected in 1 patient (16.7\%), second level - in 2 patients (33.3\%), third level in 3 patients (50\%). We obtained the following data (Table 2) when determining the degrees of inclination of the impacted teeth axes in three planes (Table 2).

5 patients (83.3\%) with 1 degree of violations of the angle of inclination of the impacted tooth longitudinal was detected in the coronary and axial planes; 2 degree in the coronary and axial planes-1 patient (16.7\%), in the sagittal plane-2 patients $(33.3 \%)$; 3 degree disorders were detected only in the sagittal plane in 4 patients $(66.7 \%)$. Due to the application of this method in clinical practice the following things were revealed: the closer the impacted tooth is located to the spinal plane and the more parallel plane of axis of the impacted tooth to the spinal plane (Spina nasalis anterior (Sna) and Spina nasalis posterior (Snp), forecast for treatment is less favorable. In addition, the greater the angle of inclination of the impacted tooth to the spinal plane, the less forecast for treatment is favorable. Thus, when the impacted tooth is located at the 1 st level of location, the time of comprehensive treatment will be 16-18 months. When the tooth is located at the 2nd level-it will take 18-24 months; at the 3rd level-24-32 months [10].

\section{Clinical Case}

Patient 0, 13 years old, complained about absence of an upper central incisor in the dental arch, vestibular position of an upper canine on the left, and crowding of teeth on the upper and lower jaws. Clinical examination was carried out according to standard methods. Examination of the oral cavity revealed the absence of tooth 1.1 (Figure 2).

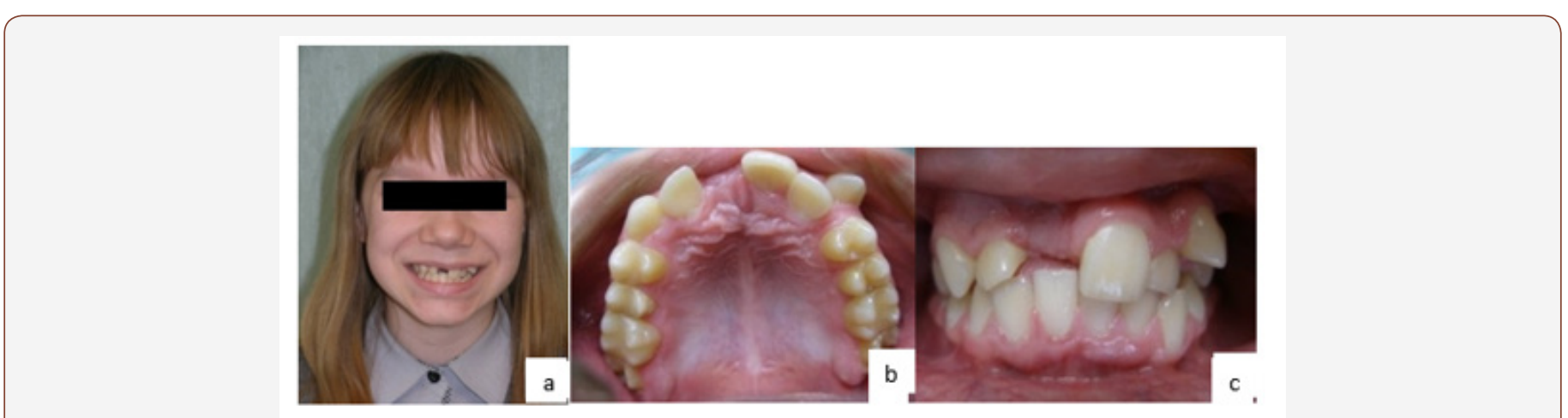

Figure 2: Photo of patient $\mathrm{O}, 13$ years old with impacted tooth 1.1 before orthodontic treatment: patient's face (a), Upper dentition from (b), Closure of dentition front view (c).

An X-ray examination of the patient included orthopantomography, telerentgenography of head in lateral projection, using Dolphin Imaging software to analyze cephalometric radiographs and cone beam computed tomography of the tooth 1.1. The study of orthopantomogram was carried out according to the method of Yu I Zhigurt. The location level of the impacted tooth 1.1 was determined (Figure 3), as well as the angle of inclination of its longitudinal axis (Figure 4). We noted three levels of the impacted teeth location in the sagittal plane on CT scan of the upper jaw according to our method. The 3rd level of the impacted tooth 1.1 location was determined (Figure 5).

Then, we measured angles of inclination of the impacted tooth 1.1 in three mutually perpendicular planes (Figure 6). 


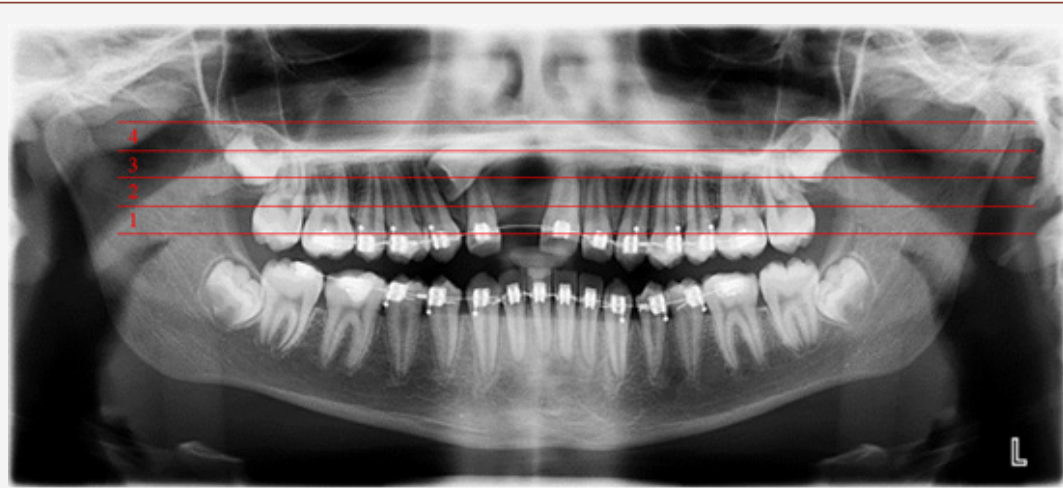

Figure 3: Location of the impacted tooth 1.1 of patient $\mathrm{O}, 13$ years old, before orthodontic treatment (3rd level according to Zhigurt UI).

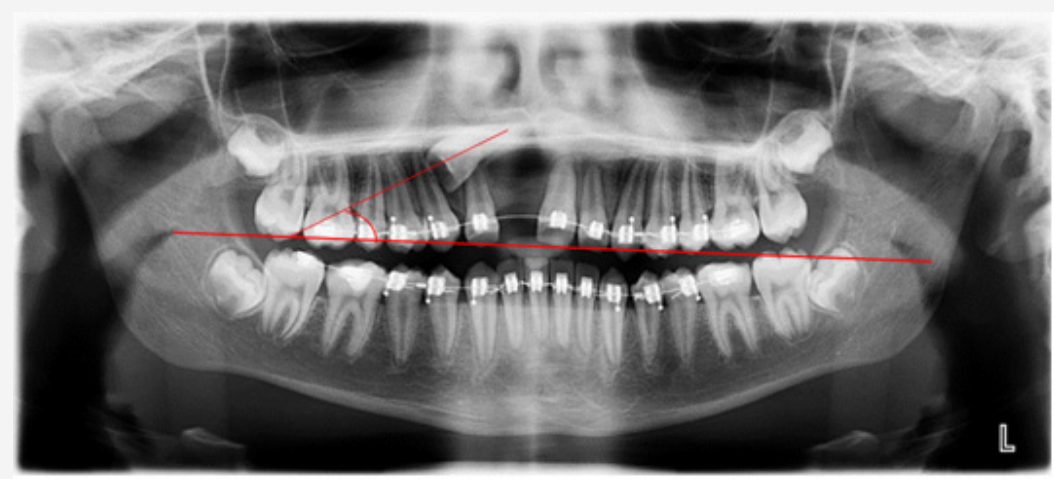

Figure 4: Location of the impacted tooth 1.1 of patient $\mathrm{O}, 13$ years old, before orthodontic treatment (angle of inclination of tooth is $30^{\circ}$ ).

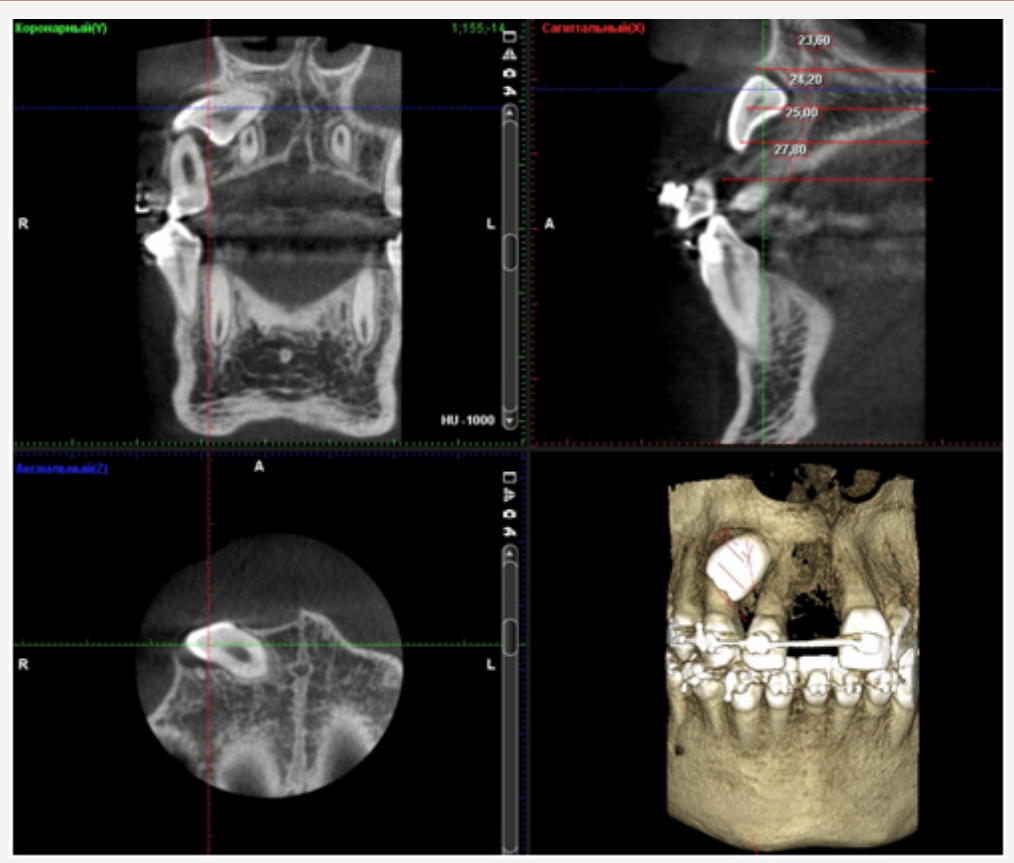

Figure 5: Location of the impacted tooth 1.1 of patient $\mathrm{O}, 13$ years old, in three projections before orthodontic treatment (3rd level of the tooth 1.1 location).

As a result of measurements, we obtained the following angles: in the coronary plane- $56.33^{\circ}$, in the sagittal plane- $129.88^{\circ}$, in the axial plane-57.93 ${ }^{\circ}$. Then, we compared the obtained data with the data in (Table 1). Angle of inclination of the impacted tooth 1.1: in the coronary plane- 2 degree; in the sagittal plane-3 degree; in the axial plane- 2 degree. Considering obtained data the diagnosis was: malocclusion class II, impaction and transposition of the tooth 1.1, crowding of teeth on the upper and lower jaws of 2-3 degrees, vestibular position of the tooth 2.3 .

Patient underwent comprehensive surgical and orthodontic treatment (Figure 7). We made one more cephalometric x-ray at the stage of orthodontic treatment with its analysis in Dolphin Imaging (Figure 8). X-ray examination was performed again after the orthodontic treatment (Figures 9,10). Comprehensive treatment of patient 0 lasted 2 years and 5 months. 


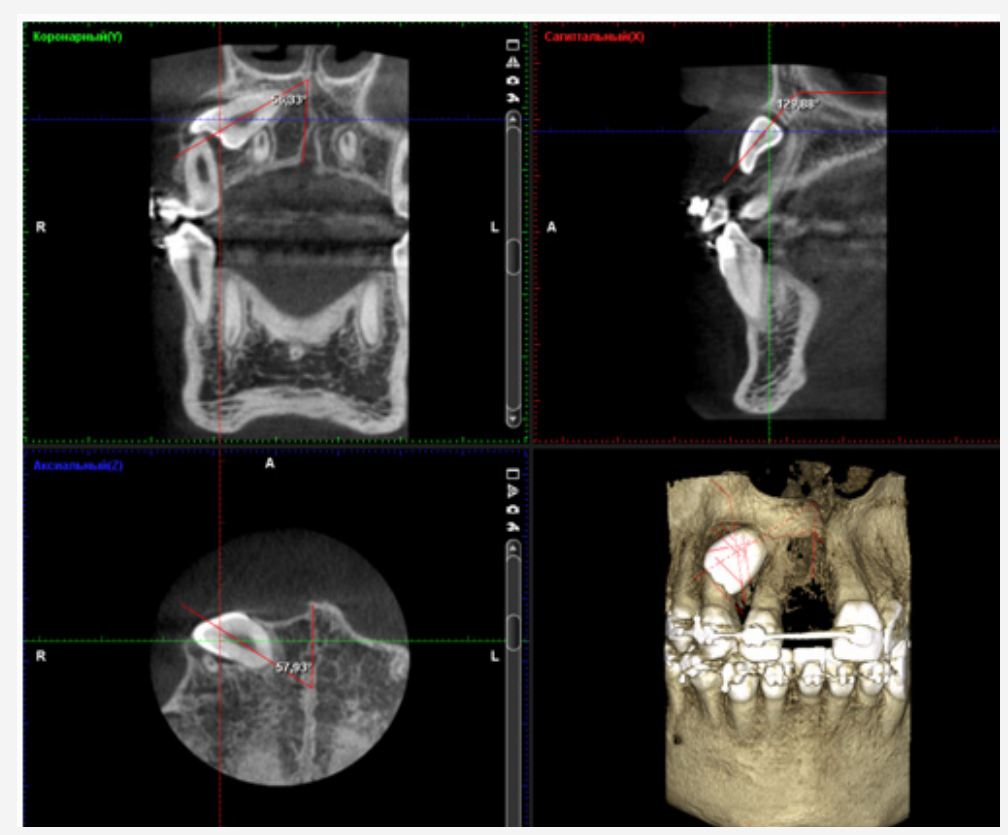

Figure 6: Determination of angles of the impacted tooth 1.1 inclination: in the coronary plane- $56.33^{\circ}$, in the sagittal plane $-129.88^{\circ}$, in the axial plane-57.93 ${ }^{\circ}$.
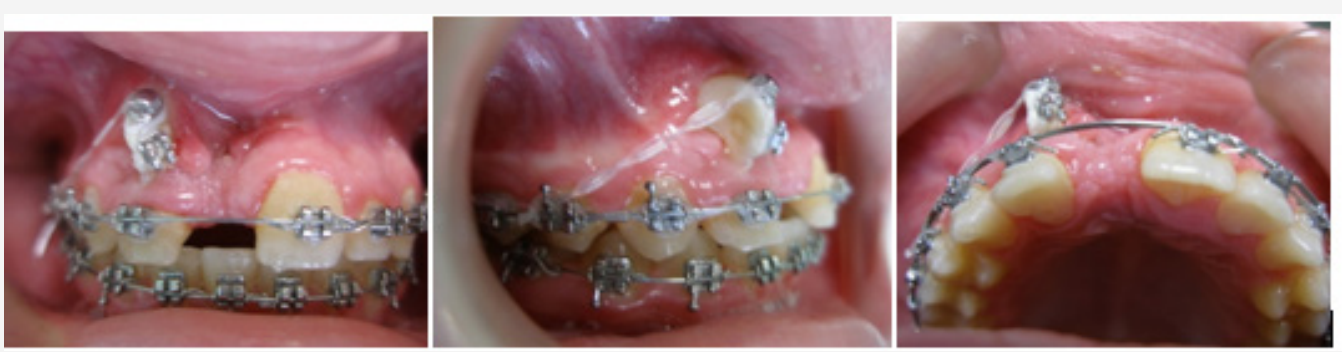

Figure 7: Photo of dentition of patient $\mathrm{O}, 14$ years old, at the stage of orthodontic treatment.

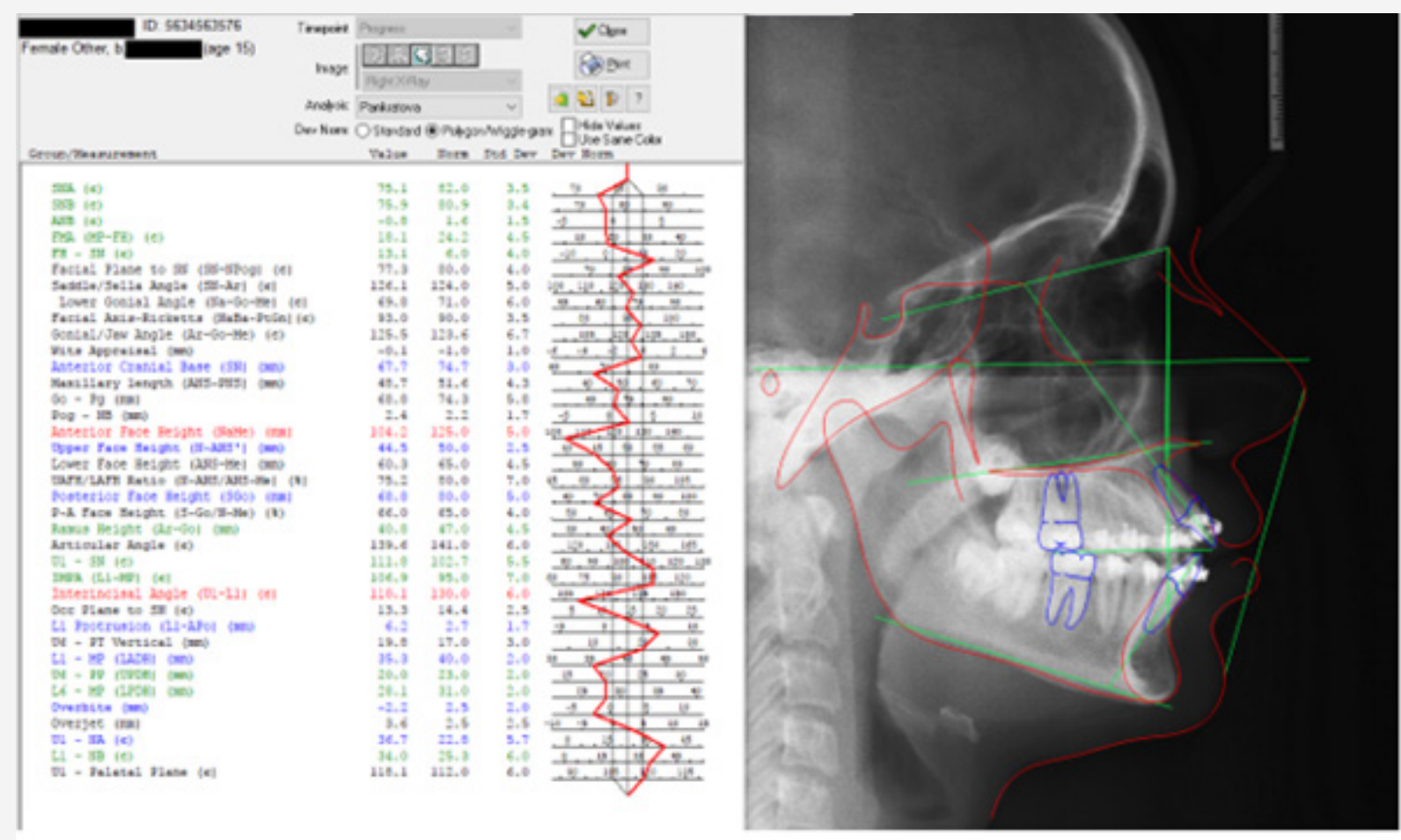

Figure 8: Cephalometric x-ray image of head in lateral projection of the patient $\mathrm{O}, 15$ years old with analysis using Dolphin Imaging software (USA) at the stage of orthodontic treatment. 


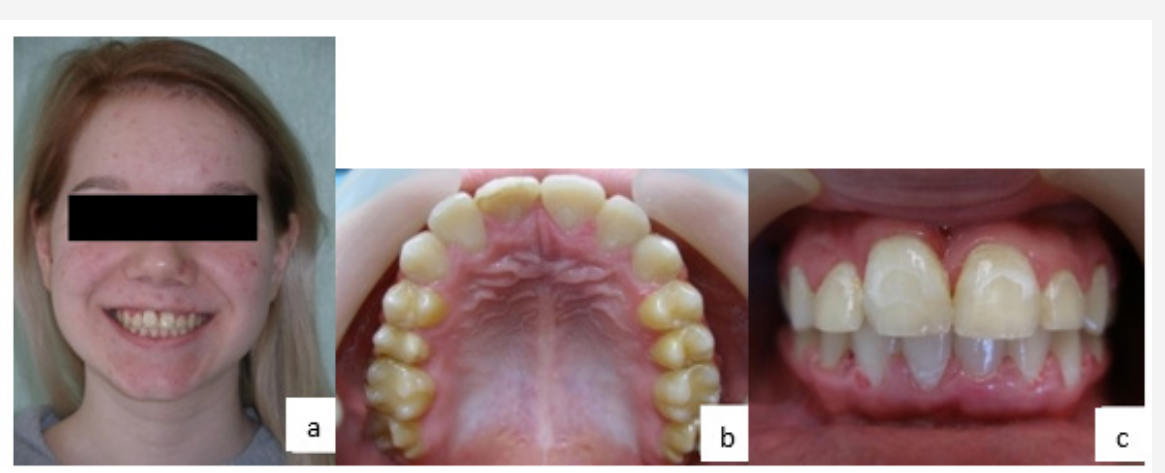

Figure 9: Photo of patient O, 15 years after orthodontic treatment (Patient's face (a), Upper dentition (b), Closure of dentition (c).

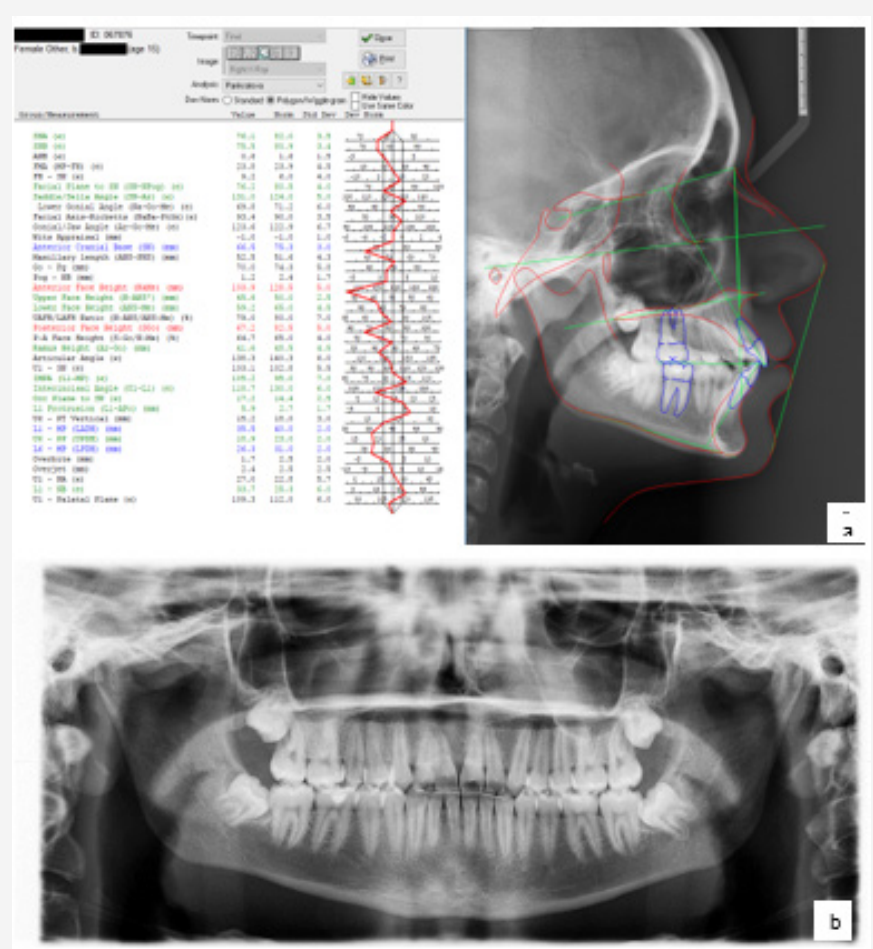

Figure 10: Cephalometric $x$-ray image of head in lateral projection of the patient $O, 15$ years old with analysis using Dolphin Imaging software (USA) after orthodontic treatment-a; location of the impacted tooth 1.1 after orthodontic treatment-b.

\section{Conclusion}

We have developed a method for determining the location of impacted teeth (incisors, canines) of the upper jaw using data from cone beam computed tomography (registration number of patients of the Russian Federation No. 2017136914 dated 10.19.2017). The given image allows us to analyze the state of elements of upper jaw from all sides without distortion. Therefore, it allows us to determine the level of impacted tooth location, the angle of inclination of its longitudinal axis more accurately.

Diagnosis of the impacted upper canines and incisors is insufficient only by the orthopantomogram of jaws. A new diagnostic method is good at planning the comprehensive treatment. Orthodontists can use this method for more accurately determining the degree of the impacted tooth location, the direction of the elastic traction in accordance with the measured angles of inclination of the impacted tooth and calculate the approximate duration of treatment.

\section{Acknowledgement}

None.

\section{Conflict of Interest}

No conflict of interest.

\section{References}

1. Arsenina OI, Proskokova SV, Sapezhnikova SA (2010) Modern methods of examination of patients with impacted teeth. Ortodontiya №1.

2. Postnikov MA (2009) Perfection of methods of orthodontic treatment of patients with impacted teeth. Pediatric Dentistry and Prevention 6(2): 30-36.

3. Fournier A, Turcotte JY, Bernard C (1982) Orthodontic considerations in the treatment of maxillary impacted canines. Am J Orthod 8(3): 236239.

4. Volchek DA (2007) Optimization of treatment of patients with impacted canines on the maxilla. Avtoref dis kand med nauk p: 1-43.

5. Postnikov MA, Stepanov GV, Seregin AS, Kirilin MM, Ulyanova LG (2017) Perfection of methods of diagnosis and treatment of patients with impacted teeth. Pediatric Dentistry and Prevention 16(61): 28-31. 
6. Zhigurt UI (1994) Planning and prognosis of treatment of impacted teeth. Avtoref dis kand med nauk Stomatologiya.

7. Vakushina EA, Bragin EA (2004) Using computed tomography in diagnostics and treatment of impacted teeth. Ortodontiya Moscow №2.

8. Aydin U, Yilmaz HH, Yildirim D (2004) Incidence of canine impaction and transmigration in a patient population. Dentomaxillofac Radiol 33(3): 164-169.
9. Becker A (2012) Orthodontic treatment of impacted teeth.

10. Chaushu S, Becker A, Zeltser R, Vasker N, Chaushu G (2004) Patients perceptions of recovery after surgical exposure of impacted maxillary teeth treated with an open-eruption surgical orthodontic technique. European Journal of Orthodontics №6. 